\title{
Dynamique de lutte contre la stigmatisation des femmes au Burundi
}

\author{
Docteur Jean de Dieu Ndikumana, \\ Enseignant-chercheur à l'École Nationale d'administration \\ Professeur visiteur à l'Université lumière de Bujumbura et à Bujumbura \\ International University
}

Doi:10.19044/esj.2020.v16n29p155 URL:http://dx.doi.org/10.19044/esj.2020.v16n29p155

\section{Résumé}

Cette réflexion avait pour objectif de démontrer la dynamique du cadre légal et règlementaire ainsi que le rôle de l'administration à la base dans la lutte contre la stigmatisation de la femme infertile. Pour asseoir ses démonstrations, l'étude utilise la méthodologie de la recherche documentaire. Celle-ci a permis de chercher et d'identifier les documents pertinents pour développer la thématique et extraire des informations permettant de répondre à l'objectif de l'étude. Cette méthodologie a servi aussi à l'exploitation du cadre légal national et des conventions et déclarations internationales traitant des questions relatives à la discrimination de la femme. Il ressort des analyses faites que le Burundi a manifesté sa volonté de combattre l'exclusion de la femme en souscrivant aux conventions internationales et en mettant en place un cadre national réglementaire et institutionnel y relatifs. Mais en dépit de l'existence de cet engagement, la stigmatisation de la femme infertile suit toujours son cours dans la culture burundaise. L'étude soutient que cette persistance de l'attitude méprisante qui dévalorise la personnalité de la femme victime et qui crée en elle le sentiment d'inutilité est due aux faiblesses du cadre légal qui traite la question incidemment. En se servant de l'examen de la réglementation et des organes mis en place par les pouvoirs publics pour veiller à la protection de la femme, cette réflexion démontre que l'administration à la base pourrait jouer un rôle fondamental pour accroitre la protection des femmes souffrant de la discrimination pour cause d'infertilité. Leurs interventions efficaces permettraient de réduire les barrières coutumières qui handicapent la dénonciation des cas de stigmatisation chez la femme infertile. C'est ainsi que l'étude suggère de créer des centres d'écoute et d'élargir le mandat des structures existantes au niveau communal pour y inclure la prise en charge des femmes victimes de stigmatisation. Pour accroitre l'efficacité des centres ainsi créés ou structures existantes, cette analyse propose de développer des mécanismes d'alerte reposant sur des 
indicateurs d'intervention préétablis.

Mots clés : Femme infertile, stigmatisation, cadre réglementaire, efforts communautaires, Burundi

\title{
Fighting Against the Stigmatization of Women in Burundi
}

\author{
Docteur Jean de Dieu Ndikumana, \\ Enseignant-chercheur à l'École Nationale d'administration \\ Professeur visiteur à l'Université lumière de Bujumbura et à Bujumbura \\ International University
}

\begin{abstract}
This reflection aimed to demonstrate the dynamics of the legal and regulatory framework as well as the role of grassroots administration in the fight against the stigmatization of infertile women. To back up its demonstrations, the study uses the methodology of documentary research. This made it possible to search and identify relevant documents to develop the theme and extract information to meet the objective of the study. This methodology has also been used to exploit the national legal framework and international conventions and declarations dealing with issues relating to discrimination against women. The analyzes carried out show that Burundi has shown its willingness to combat the exclusion of women by subscribing to international conventions and by putting in place a national regulatory and institutional framework relating to it. But despite the existence of this commitment, the stigmatization of infertile women continues to run its course in Burundian culture. The study argues that this persistence of the contemptuous attitude which devalues the personality of the female victim and which creates in her the feeling of worthlessness is due to the weaknesses of the legal framework which deals with the issue incidentally. By using the review of the regulations and bodies set up by the public authorities to ensure the protection of women, this reflection demonstrates that the grassroots administration could play a fundamental role in increasing the protection of women. suffering from discrimination because of infertility. Their effective interventions would make it possible to reduce the customary barriers that handicap the denunciation of cases of stigmatization among infertile women. This is how the study suggests creating listening centers and broadening the
\end{abstract}


mandate of existing structures at the municipal level to include care for women victims of stigmatization. To increase the efficiency of the centers thus created or existing structures, this analysis suggests developing alert mechanisms based on pre-established intervention indicators.

Keywords: Infertile woman, stigma, regulatory framework, community efforts, Burundi

\section{Introduction}

Quand l'enfant vient à manquer dans un couple, la femme est tenue pour responsable de cette infertilité par son mari. Le mariage rime avec la procréation et une union sans enfants n'a pas de sens et sa raison d'être est souvent remise en cause. Dans bon nombre de cas, la situation d'infertilité conduit à l'exclusion de la femme; celle-ci pouvant être délaissée pour ce dont elle n'est pas du tout responsable avec notamment comme effet, son indexation et, partant, la complication des relations conjugales et sociales (Navas, 1977). La question de l'infertilité, et partant son incidence négative surtout envers la femme taxée d'infertile par la communauté, pose un réel problème de cohésion familiale et sociale. En effet, la stigmatisation ayant pour cause l'infertilité est tellement d'une grande sensibilité et d'une discrétion pour les époux et les familles, qu'il faut éviter de la laisser à eux seuls. La raison de cette étude est justement d'analyser les termes dans lesquels se manifeste cette stigmatisation afin de proposer des éléments de réponse pour remédier à ce fléau social. Cette démarche aboutit à la question de savoir quelles sont les actions que l'administration à la base peut déployer pour contrer le phénomène de stigmatisation envers la femme infertile, ou à tout le moins en atténuer les effets? Cette étude considère que l'administration à la base est un acteur incontournable pour lutter contre cette stigmatisation. Non seulement elle est plus proche des victimes, mais elle dispose aussi d'un cadre légal qui peut être réaménagé pour prendre en compte ce problème social. Une analyse de ce phénomène permet son appréhension et constitue une base pour formuler des propositions de nature à favoriser sa meilleure prise en charge ainsi qu'une implication active de l'administration à la base reconnue plus efficiente pour gérer les affaires de proximité (Kagisye, 2009). Pour aborder cette thématique de dynamique de lutte contre la stigmatisation de la femme au Burundi, trois axes sont développés. L'étude expose d'abord l'introduction (I), la recension des écrits (I.1), des analyses au sujets de la stigmatisation (I.2) et l'objectif de l'étude. La partie suivante comporte la méthodologie (II) qui précise les techniques de recueil des données (II.1) et leurs méthodes d'analyse (II.2). Ensuite, des résultats (III) sont dégagés à travers l'analyse du cadre normatif de protection de la femme infertile stigmatisée (III.1). Sous cet angle, l'examen porte sur le cadre conventionnel (III.1.1) et le cadre légal national 
(III.1.2). L'analyse portera aussi sur la Constitution de 2018 (III.1.2.1) et d'autres institutions et entités locales. Il s'agira de relever les compétences dévolues aux institutions dans la loi communale (III.1.2.2) dont notamment la commune (III.1.2.2.1), le conseil communal (III.1.2.2.2), l'administrateur communal (III.1.2.2.3) et le chef de quartier/colline (III.1.2.2.4). Cette démarche permettra de mettre en exergue le processus de stigmatisation de la femme infertile (III.2) et de constater la stigmatisation consécutive de la femme infertile (III.2.1). Enfin, les développements montrent qu'il y a des efforts en cours pour combattre le phénomène de la stigmatisation de la femme infertile (III.3) à travers l'administration burundaise (III.3.1) et les mouvements associatifs (III.3.2) parmi lesquels en découlent des propositions d'amélioration des dispositifs mis en place par rapport à la protection de la femme infertile stigmatisée (III.4).

\section{I.1. Recension des écrits}

À l'échelle mondiale, l'infertilité constitue non seulement un problème majeur de santé, mais aussi un défi social avec des retombées négatives aussi bien sur les couples et la communauté que sur la femme qui en est victime (Ombelet, 2012). L'infertilité est définie par l'OMS comme l'incapacité d'un couple à parvenir à concevoir et à mener une grossesse à terme à un an ou plus de rapports sexuels réguliers et non protégés pour les femmes de moins de 35 ans et après 6 mois pour les femmes de plus de 35 ans (OMS 2014). Le problème d'infertilité s'accompagne souvent de la stigmatisation qui comporte des effets néfastes sur les personnes affectées (Courtwright, 2013). La stigmatisation constitue un phénomène social qui conduit au rejet ou à la mise à l'écart d'une personne ou d'un groupe de personnes qui sont perçus comme allant à l'encontre des normes culturelles du groupe ou de la société à laquelle elles appartiennent. La stigmatisation est un processus dynamique de dévaluation qui discrédite significativement un individu aux yeux des autres (Erving, 1963). Il sied de noter que l'infertilité ne doit pas être envisagée uniquement dans le cadre des personnes engagées dans les liens d'un mariage. La question peut se poser pour toute personne en âge de procréer et qui attend un enfant à partir de deux ans et vivant des rapports sexuels non protégés.

L'infertilité est devenue une préoccupation mondiale, car elle affecte plus de 180 millions de couples dans le monde. Un couple sur dix consulterait dans une structure de soins pour cause d'infertilité (OMS, 2016). L'OMS indique que sur les 180 millions de couples touchés par l'infertilité dans le monde, la moitié vit en Afrique subsaharienne avec une prévalence pouvant augmenter jusqu'à $33 \%(\mathrm{OMS}, 2013)$. On estime que ce taux d'infertilité, qui varie d'une région à une autre, est globalement élevé (Larsen, 1994). Même si la médecine permet aujourd'hui de résoudre une grande partie des cas d'infertilité, certaines situations restent insolubles, puisqu'environ 48,5 à 72,4 
millions soit 7-9\% des couples dans le monde ont un problème non résolu d'infertilité (Olatunjla, 2003). À ce stade, l'infertilité suscite des questionnements au sein même des couples, dans les familles et dans l'entourage. Des analyses démontrent que dans toutes les cultures du monde, donner la vie est l'acte le plus important pour la perpétuité de la race humaine (Navas, 1977). Dans la conception de nombreux Africains, la fécondité est un signe de bénédiction qui fait des couples, des êtres complets ayant atteint une maturité complète et, de ce fait, la procréation est considérée comme l'objectif premier du mariage (Navas, 1977). Dans la société burundaise, la fécondité occupe une place centrale dans la stabilité et la survie du couple. Elle est perçue comme « la valeur fondamentale de cellule familiale. L'enfant apparait comme la pierre angulaire de tout l'édifice familial burundais » (Navas, 1977). Dans le contexte burundais, la femme endosse la responsabilité, à tort ou à raison, de l'absence d'un enfant dans un couple. Face à la stigmatisation, la femme infertile se sent moins femme, et se retrouve donc dans une catégorie autre, celle des infertiles (Dyer et al, 2005).

\section{2 Limites des analyses au sujet de la stigmatisation}

Les écrits répertoriés sur l'infertilité mettent l'emphase sur la description de l'existence du phénomène de stigmatisation due à l'infertilité de la femme en Afrique. C'est cette même démarche descriptive que peu d'auteurs burundais qui traitent le sujet empruntent. Les solutions que proposent la littérature sont difficilement généralisables au regard de la spécificité de chaque pays et de la diversité des traditions coutumières.

Le cadre légal mis en place au Burundi pour protéger les femmes contre toutes formes de discrimination se limite souvent sur la promotion du genre dans son ensemble. Il n'existe pas de réglementation spécifiquement dédiée à la lutte contre la stigmatisation que rencontrent les femmes infertiles au sein de leurs ménages et aussi dans la communauté où elles vivent. C'est donc un cadre légal qui traite incidemment la question et cela fait que le phénomène continue à s'accentuer. Par ailleurs, ledit cadre légal manque des textes d'application pouvant faire ressortir le caractère contraignant chaque fois qu'il se présente un cas de stigmatisation dans la communauté. Par voie de conséquence, l'administration locale qui est un acteur incontournable pour lutter contre le phénomène mène des actions qui s'inscrivent dans le cadre général de protection de la femme. Par ailleurs, la coutume burundaise reste une limite quant à l'interprétation et l'application des lois et conventions que le gouvernement a mises en place.

\section{I.3 Objectif de l'étude}

Cette étude analyse le phénomène de stigmatisation dans la perspective du cadre institutionnel et légal du Burundi en vue de suggérer des propositions 
visant à améliorer l'action de l'administration locale pour combattre la stigmatisation de la femme infertile.

\section{Méthodologie}

Cette partie traite des techniques de recueil de données utilisées ainsi que leurs méthodes d'analyse.

\section{II.1 Techniques de recueil des données}

Étant donné que la méthodologie dans ce travail est documentaire, la technique de collecte des données consistera à identifier les sources documentaires à exploiter. Pour l'essentiel, il s'agira de sélectionner les documents pertinents incluant les livres, les articles des revues scientifiques et les divers rapports des organisations et institutions. Plusieurs types de ressources à savoir les catalogues de bibliothèques, les bases de données bibliographiques ainsi que les ressources du Web permettront de visiter quelques sites en utilisant des moteurs de recherche spécialisés. D'autres éléments d'informations seront recueillis en rassemblant la réglementation nationale dans le domaine de la protection des femmes ainsi que les conventions internationales sur la lutte contre la discrimination de la femme.

\section{II.2 Méthodes d'analyse des données}

Ayant déjà rassemblé les informations émanant des diverses sources, la méthode d'analyse consistera à les compiler et à les recouper afin d'en extraire les plus pertinentes pour traiter la problématique de cette étude. Par après, il s'agira d'en effectuer l'analyse au regard de nos objectifs de recherche et d'en dégager les grandes idées qui guideront la rédaction.

\section{Résultats}

Les résultats que présente cette étude sont dégagés de l'examen des textes destinés à protéger la femme et à lutter contre toute forme de discrimination qui la vise afin d'en dégager la portée et les limites. Mais ces résultats sont aussi dérivés de l'analyse du processus de stigmatisation de la femme infertile pour en faciliter la compréhension. Une autre partie des résultats concerne l'analyse des efforts que les pouvoirs publics burundais déploient pour combattre le phénomène de la stigmatisation de la femme infertile. L'étude aboutit à la conclusion que l'administration locale est mieux indiquée pour l'effectivité de la lutte contre la stigmatisation de la femme infertile et formule des propositions d'améliorations des dispositifs mis en place. 


\section{III.1 Analyse du cadre normatif de protection de la femme infertile}

Dans cette partie, il s'agit d'analyser la réglementation burundaise dédiée à la protection de la femme infertile ainsi que les conventions que le Burundi a ratifiées pour lutter contre toute sorte de discrimination dirigée contre elle.

\section{1.1 Cadre conventionnel}

L'adhésion du Burundi à l'ONU a eu pour corollaire d'incorporer la Déclaration universelle des Droits de l'Homme dans son cadre légal interne. Cet instrument consacre en son article 7 le droit à la protection contre toute forme de discrimination et la stigmatisation en est une. Dans sa philosophie législative, protéger et garantir la dignité de la femme constituent le leitmotiv de l'appareil législatif burundais. Le pays a ratifié le pacte international relatif aux droits économiques, sociaux et culturels (loi no 11/008 du 14 mars 1990). En son article 3, ce texte consacre la jouissance de tous les droits économiques en énonçant : «Les États parties au présent Pacte s'engagent à assurer le droit égal qu'ont l'homme et la femme au bénéfice de tous les droits économiques, sociaux et culturels qui sont énumérés dans le présent Pacte» (Sebudandi et Ndikumana, 2012).

Le Burundi est respectivement membre de la Convention sur l'Élimination de toutes les formes de Discrimination à l'égard des Femmes (CEDEF) (ratifiée le 8 janvier 1992) et du protocole additionnel à la Charte africaine des droits de l'homme et des peuples relatifs aux droits de la femme en Afrique signé le 13 novembre 2001. Au sens de la CEDEF, «la discrimination à l'égard de la femme vise toute distinction, exclusion ou restriction fondée sur le sexe qui a pour effet ou pour but de compromettre ou de détruire la reconnaissance, la jouissance ou l'exercice par les femmes, quel que soit leur état matrimonial des droits de l'homme et des libertés fondamentales dans le domaine politique, économique, social, culturel et civil, et dans tout autre domaine ». L'article 2 de la CEDEF engage les États parties à «poursuivre par tous les moyens appropriés et sans retard une politique tendant à éliminer la discrimination à l'égard des femmes ».

L'adhésion à ces différentes conventions internationales et la souscription à certaines de ces déclarations suscitées ont eu pour effet leur incorporation dans le droit positif burundais. Cette partie de la législation burundaise démontre une volonté manifeste des autorités de la République de traiter également, et cela au haut niveau, tous les citoyens sans discrimination basée sur le sexe. Le cadre légal national s'oriente dans une certaine mesure, dans cette direction favorable à la protection des femmes contre toute sorte de discrimination. Évidemment on peut déplorer le décalage qui existe entre la coutume burundaise et l'évolution des droits de la femme aussi bien à l'échelle nationale qu'internationale. 


\section{III.1.2 Cadre légal national}

Le cadre légal sera analysé sous l'angle des dispositions de la Constitution de 2018, de la loi communale et des dispositions régissant la répression des violences basées sur le genre.

\section{III.1.2.1 La Constitution de 2018}

Le Burundi a manifesté, et c'est toujours le cas, son attachement à régler la question de l'injustice sociale. Comme les précédents développements le montrent, il a, et continue, de poser les bases juridiques pour lutter contre la discrimination basée sur tout type d'exclusion sociale.

La Constitution burundaise de juin 2018 pose et défend le principe de l'égalité entre tous les citoyens. En son article 13, ce texte fondamental prône l'égalité entre tous les Burundais et la jouissance des mêmes droits par tous les citoyens sans distinction basée notamment sur le sexe (Constitution de 2018). La même Constitution dispose que « les droits et devoirs proclamés et garantis, entre autres, par la Déclaration universelle des droits de l'homme, les Pactes internationaux relatifs aux droits de l'homme, la Charte africaine des Droits de l'Homme et des peuples, la Convention sur l'élimination de toutes les formes de discrimination à l'égard des femmes et la Convention relative aux droits de l'enfant font partie intégrante de la Constitution de la République du Burundi» (Constitution de 2018). L'article 22 de la même constitution ajoute que «nul ne peut être l'objet de discrimination du fait notamment de son origine, de sa race, de son ethnie, de son sexe $[\ldots] »$.

\section{III.1.2.2. Des compétences dévolues aux institutions dans la loi communale}

Selon l'article 1 de cette loi, la commune est une collectivité territoriale décentralisée, dotée de la personnalité juridique, de l'autonomie organique et financière. Cette organisation administrative confère diverses compétences à la commune que celle-ci peut déployer pour résoudre les questions sociales auxquelles sont confrontés ses habitants.

\section{III.1.2.2.1. La commune}

La loi communale confère à la commune des compétences en matière sociale. Aux termes de l'article 6, cette loi énonce que la commune constitue la base économique et sociale de la population établie sur son territoire. Pour pleinement jouer ce rôle, ses organes doivent veiller constamment à promouvoir le développement communautaire sur tous les plans et travailler pour améliorer le sort de ses habitants (Loi ${ }^{\circ} 1 / 33$ du 28 novembre 2014 portant Révision de la Loi n ${ }^{\circ}$ 1/02 du 25 janvier 2010 portant Organisation de l'Administration communale).

Relativement aux organes d'administration et de gestion de la 
commune ainsi que de ses entités, la loi communale contient des dispositions qui, sans répondre directement à la question de lutte contre la stigmatisation de la femme infertile, constituent au moins des références pouvant leur permettre de se saisir de la question et prendre des initiatives pour s'occuper des personnes victimes de la stigmatisation de la femme infertile (Loi $\mathrm{n}^{\circ} 1 / 33$ du 28 novembre 2014 portant Révision de la Loi n ${ }^{\circ}$ 1/02 du 25 janvier 2010 portant Organisation de l'Administration communale).

\section{1.2.2.2 Le conseil communal}

L'article 14 confère des pouvoirs à cet organe pour formuler ses avis sur toutes les affaires notamment relevant du social. En son article 15, la loi communale exige au conseil communal d'organiser au moins 2 fois par an des rencontres ouvertes au conseil des collines et aux représentants des associations œuvrant dans la commune pour les informer de manière transparente sur la situation notamment sociale prévalant dans la commune. La tenue de telles assises des organes sociaux constitue donc un moment privilégié pour inscrire les questions de stigmatisation à l'agenda des discussions. Qui plus est, cette exigence que pose la loi confère aux associations qui luttent pour l'amélioration des droits humains une tribune d'expression pour proposer toute solution au problème de stigmatisation. Il y a lieu de noter également que conformément à l'article 22 de la loi communale, le conseil communal constitue en son sein des commissions permanentes ou temporaires de manière à couvrir tout le champ de compétence de la commune. Ainsi formulée, cette disposition laisse de l'espace pour des initiatives que les autorités à la base peuvent prendre tout en restant dans la légalité (Loi n ${ }^{\circ} 1 / 33$ du 28 novembre 2014 portant Révision de la Loi n ${ }^{\circ}$ 1/02 du 25 janvier 2010 portant Organisation de l'Administration communale).

Le Manuel communal de Procédures administratives et financières amplifie l'autonomie de gestion du conseil communal. Il y est prévu, la création des commissions des Affaires sociales et du Genre chargées notamment de l'assistance sociale, de la protection et de la promotion des groupes vulnérables, de la santé, de la culture et de la promotion de la femme. Le cadre légal est ainsi flexible et offre aux acteurs sociaux un cadre de discussion, mais aussi d'action sur tous les enjeux sociaux dans la commune. Le défi dans la lutte contre la stigmatisation n'est donc pas le manque du cadre d'action, mais plutôt d'initiatives réalistes pouvant aboutir aux résultats tangibles. Ceci se comprend dans la mesure où le phénomène de stigmatisation est complexe ; ce qui complique son appréhension par les autorités à la base peu sensibilisées et surtout imbues de la coutume locale qui constitue leur référence de prédilection dans la prise des décisions. 


\section{III.1 2.2.3 L'Administrateur Communal}

Les prérogatives pertinentes en lien avec la lutte contre la stigmatisation de la femme infertile se retrouvent aux articles 25, 26, et 30 de la loi communale. L'article 25 précise notamment que cette autorité communale coordonne toutes les actions de développement socioéconomique qui se mènent sur tout le territoire de la commune. Ce faisant, elle offre à l'administrateur communal l'opportunité de revoir ce qui est proposé et d'en superviser l'exécution. Autrement, l'administrateur communal dispose d'une compétence élargie qu'il peut déployer pour encadrer efficacement la lutte contre la stigmatisation de la femme infertile.

L'article 26 quant à lui, dispose que l'Administrateur Communal exerce dans les limites territoriales de son ressort un pouvoir général de police et qu'il prend à cet effet toute mesure de police qu'il juge utile au maintien de l'ordre et de la sécurité publics. Il s'agit d'un pouvoir à double facette. Non seulement l'administrateur peut réglementer dans sa commune, mais il peut aussi sanctionner les agissements qui perturbent la quiétude de sa population. Or, la stigmatisation produit parfois des effets qui troublent l'ordre public et perturbent l'harmonie familiale et la cohésion du tissu social. L'administrateur peut user de ces prérogatives pour proposer des mécanismes de lutte contre la stigmatisation et les dégâts qui en résultent.

$\mathrm{Au}$ sujet de l'article 30, la loi précise qu'il coordonne toutes les activités des services œuvrant dans sa commune. Il est assisté par un conseiller technique chargé notamment des affaires sociales. Si le problème de stigmatisation se pose dans la commune, ce cadre technique qui accompagne l'administrateur communal dans sa mission de coordination pourrait s'en saisir et proposer des voies de solutions pour y remédier (Loi n ${ }^{\circ} 1 / 16$ du 25 mai).

\section{III.1.2.2.4 Le chef de quartier/colline}

La loi communale donne à ce responsable à la base la mission d'assurer au sein du quartier/colline l'arbitrage, la médiation, la conciliation ainsi que le règlement des conflits sociaux (article 46,2 $2^{\circ}$. Cette compétence que ces organes héritent de cette loi fait d'eux des partenaires incontournables dans le système de règlement pacifique des conflits au Burundi. Dans la tradition burundaise, il existe une institution des sages (Abashingantahe) qui tranche des conflits à l'échelon collinaire. Ces notables du village font donc de la médiation sur tout conflit social qui surgit dans la communauté. Leurs solutions n'ont pas de caractère contraignant, mais les parties les mettent souvent en application. Ils sont donc les premiers à connaitre de toute nature de conflit y compris ceux qui naitraient autour de la question de stigmatisation. Il pourrait s'agir des problèmes de répudiation de divorce ou de violence conjugale. Mais jusque là avec ces notables, il s'agit de trancher des conflits 
ayant un caractère de nature physique. Les conflits ayant un caractère psychologique ne sont pas pris en charge. Dans cette loi, il est prévu que le chef de colline ou de quartier soit l'animateur de la paix sociale et du développement dans sa circonscription. Cette attribution offre à ces responsables à la base la possibilité de sensibiliser les membres de leur communauté sur la thématique de stigmatisation de la femme infertile. C'est ainsi que la loi permet à ces autorités à la base le pouvoir d'organiser au moins une fois par trimestre une réunion ouverte à tous les habitants du quartier/colline pour analyser notamment les questions d'ordre sociales prévalant dans le quartier/colline. En effet, animer une sensibilisation sur un phénomène aussi complexe que la stigmatisation exige un niveau de compréhension moins à la portée des animateurs à ce niveau local. Certes le problème peut être abordé, mais de façon superficielle.

\section{III.2 Processus de stigmatisation de la femme infertile}

Si certaines femmes tombent enceintes au premier essai, d'autres ne le seront pas ou attendront des années. Cette situation affecte profondément la vie du couple, mais surtout celle de la femme qui est souvent considérée comme la cause du problème. Au Burundi, en l'absence d'une progéniture, c'est la femme qui est pointée du doigt parfois à tort (Novas, 1977). C'est ainsi que la belle-famille commence à s'en mêler et à s'en prendre particulièrement à la belle-fille. Alors on utilise certaines expressions orales méprisant la bellefille telle que : "Yaje guhonya umuryango » (elle est venue exterminer la lignée) ou alors "Yazanywe n'ukurya gusa" (elle est venue juste pour manger). L'enfant étant considéré comme une richesse, une femme qui ne met pas au monde est donc perçue comme une malédiction pour sa belle-famille (Erving, 1963). À cet état de choses s'ajoutent la pression du voisinage et les ragots de la société. La femme se sent finalement coupable, quand bien même ce ne serait pas de sa faute, car culturellement la fertilité de l'époux est rarement mise en doute. Lorsqu'elle existe, elle est tolérée et la femme est réduite au silence lorsqu'elle ne reçoit pas l'aval de son mari pour chercher une grossesse ailleurs dont il assumera la responsabilité d'auteur. À cela se joignent la tristesse et la dépression quand la femme se retrouve dans des groupes de femmes parlant de maladies infantiles, allaitement, anniversaires, etc. Elle se sent du coup comme une intruse, une marginale (Nana et al, 2011).

La société burundaise considère le mariage comme une union dans laquelle la femme doit offrir une progéniture, un héritier à son mari (Navas, 1977). En l'absence d'enfant dans le mariage, l'union n'a pas de sens et cela se traduit souvent par une réelle exclusion de la femme. Conséquemment, elle peut être délaissée avec comme effet son indexation et, partant, la complication des relations conjugales et sociales (Navas, 1977).

En effet, la stigmatisation ayant pour cause l'infertilité déstabilise le 
couple et l'harmonie dans la communauté. Mais la femme qui en endosse la responsabilité en porte aussi des séquelles indélébiles.

L'infertilité dans le couple devient alors un véritable calvaire pour la femme. Elle est étiquetée par son mari puis sa belle-famille et par son entourage et la communauté, y compris même ceux qui se trouvent être des évolués instruits, des intellectuels (Maggio, 2006). Autrement, les auteurs de la stigmatisation se recrutent parmi toutes les couches du tissu social.

Au-delà de ces souffrances psychologiques, la femme infertile fait face au risque de répudiation, de divorce et d'accusation de sorcellerie (Bonnet, 2010).

La stigmatisation envers la femme infertile souffrant des perceptions et préjugés sociaux constitue un mal de société dû à l'ignorance qu'il convient de combattre. L'infertilité est un handicap que la victime porte sans y avoir consenti et lui en faire peser la responsabilité est une injustice à laquelle doit remédier la société. Pour qu'il en soit ainsi, la coutume devrait rattraper la volonté juridique que le pays a déjà manifestée dans le domaine de la protection des droits des femmes.

\section{III.2. Stigmatisation consécutive à l'infertilité}

Depuis la fin des années 1990, beaucoup d'études replacent l'infertilité dans son contexte social, au-delà du contexte purement clinique, bien que celui-ci demeure prépondérant. Comme l'explique Greil : « l'infertilité [est] un processus socialement construit dans lequel les individus définissent leur capacité à avoir des enfants comme problème, à définir la nature de ce problème est à élaborer un plan d'action appropriée. » (Greil, 1991).

L'OMS qui reconnaît l'infertilité comme un jeu de santé publique a établi aussi des lignes directives invitant le personnel médical à aller au-delà des diagnostics et des interventions cliniques et à porter une attention particulière aux aspects psychologiques liés aux problèmes de fertilité afin d'œuvrer pour l'amélioration de la qualité de vie des couples infertiles (Rapport de l'OMS, 2013).

D'un point de vue social, le vécu et les enjeux qui entourent l'infertilité dépendent largement des valeurs et des représentations qui gravitent autour de l'enfant et de la place qu'occupe celui-ci dans la définition de la famille. Dans la mesure où elle réfère avant tout à des couples qui désirent des enfants et qui n'arrivent pas à en concevoir, la notion même d'infertilité demeure étroitement liée aux conjonctures culturelles et historiques dans lesquelles s'inscrit la venue au monde d'un enfant (Chateauneuf, 2011).

Dans leur travail, Dyer et ses collaborateurs évoquent ce qu'ils ont appelé une stratégie de secret pour cacher l'infertilité (Dyer et al, 2005). Cette stratégie met en marche une culture du silence qui entoure bien souvent, et un peu partout en Afrique, les problèmes reproductifs, dont l'infertilité. Dupiesh 
s'est interrogée sur la possibilité d'une stigmatisation de l'infertilité envers la femme (Dupiech, 2004) en reprenant les concepts d'identités définis par Goffman dans ses travaux sur les personnes stigmatisées. Il conclut que considérer l'infertilité comme un statut, c'est faire preuve d'impartialité ; la considérer comme un problème, c'est lui conférer un caractère éminemment négatif.

Cette constatation de Dupiech est perceptible dans l'usage des termes tels qu' «incapacité à se reproduire», «impossibilité de concevoir», « inaptitude à la gestation», «non-fertilité », « défaillance corporelle », «dysfonctionnement physiologique» (Dupiech, 2004). Ces concepts dégradants ont été transposés dans le contexte burundais avec parfois une plus grande ampleur de ce qu'ils représentent comme connotation dans les autres cultures. Ainsi rencontre-t-on en langue nationale l'usage des termes comme « ingumba, intavyara » se traduisant comme l' « incapacité de reproduire ».

Les auteurs de la stigmatisation devraient se ressourcer dans cette réflexion de (Hakizimana, 2004) qui considère que « [s] e donner un enfant dépasse les compétences et les performances humaines et relève des secrets et des mystères réservés exclusivement à Imana « Dieu ». Dans l'idéalisation de la maternité, une mère «umuvyeyi » représente l'humanité et une femme stérile « ingumba » symbolise l'animalité (Hakizimana, 2004).

La stigmatisation est un phénomène social qui conduit au rejet ou la mise à l'écart d'une personne ou d'un groupe de personnes qui sont perçus comme allant à l'encontre des normes culturelles du groupe ou de la société à laquelle elles appartiennent. La stigmatisation est un processus dynamique de dévaluation qui discrédite significativement un individu aux yeux des autres (Erving, 1963).

L'attribut discriminant (stigmate) peut être d'ordre corporel (handicaps physiques, couleur de la peau, aspect et anomalies du corps, etc.) ou tenir à la personnalité et/ou au passé de l'individu (troubles du caractère, séjour passé en hôpital psychiatrique, alcoolisme et autres conduites addictives, etc.). Ces stigmates peuvent être visibles : l'individu est alors discrédité. Il est perçu comme occupant une position inférieure, ce qui modifiera la nature de ses relations. Le stigmate affecte ainsi son identité sociale, son identité personnelle et le regard qu'il porte sur lui-même (Rochedy, 2015).

Les stigmates peuvent être invisibles : l'individu est « discréditable » et son problème devient celui du contrôle de l'information à propos de son stigmate. Les conséquences les plus importantes sont la perte d'estime de soi. Celui-ci est une dévalorisation liée au sentiment de ne pouvoir jouer le même rôle social que les autres du fait de son stigmate. Cette perte développe le sentiment de culpabilité chez la victime (Rostaing, 2015). Le stigmate pour cause d'infertilité vis-à-vis de la femme burundaise fait partie de cette 
catégorie de stigmates invisibles.

Le concept de stigmatisation de Goffman illustre bien les conséquences sociales qui se déclenchent quand un individu ne peut pas répondre aux spécificités assignées à son rôle social ou à son sexe (Erving, 1963).

Cette problématique est un fardeau supplémentaire que la majorité de l'opinion s'accorde à faire peser sur la femme, alors seule responsable de l'impossibilité de continuer la lignée. L'incapacité de concevoir ou de porter à terme un enfant peut susciter des problèmes psychologiques chez la femme infertile, vu qu'elle ne peut se réaliser en tant que femme. Si elle n'enfante pas, elle sera comme si elle n'avait jamais existé. Si un homme peut continuer à vivre en se réincarnant dans un fils ou petit-fils de son frère, ceci n'est pas possible pour une femme (Nkurunziza, 2009).

L'homme, à qui la société burundaise reconnait la qualité de chef de famille, celui sur qui repose le pouvoir de perpétuer son lignage, ne peut en aucun cas être la cause de l'infertilité de son couple ; raison pour laquelle celle qui est tenue pour responsable de cet échec est la femme.

Elle se culpabilise elle-même, elle commence à se douter d'elle-même, à se qualifier de stérile, car selon la culture burundaise c'est elle qui est source de procréation, c'est elle qui conçoit (Dyer, 2010).

\section{III.3 Des efforts en cours pour combattre le phénomène de stigmatisation de la femme infertile}

Les efforts déployés pour combattre le phénomène de stigmatisation de la femme infertile peuvent s'analyser sous deux angles. D'une part, il y a les actions entreprises par l'administration du Burundi et d'autre part les interventions du mouvement associatif.

\section{III.3.1 Les efforts de l'administration burundaise}

Dans la perspective de mettre en place un cadre institutionnel adéquat dédié aux questions de droits de l'Homme, le gouvernement burundais s'est doté du Ministère de la Solidarité nationale, des Affaires sociales, des Droits de la Personne Humaine et du Genre. La protection des droits des femmes et la promotion de l'égalité font partie des innombrables missions assignées à ce ministère (art 45 du Décret no 100/007 du 28 juin 2020 portant révision du décret no 100/037 du 19 avril 2018 portant structure, Fonctionnement et Mission du Gouvernement de la République du Burundi). Au sein de ce ministère, il existe une direction générale des droits de la personne humaine, de l'éducation à la paix et la réconciliation nationale. Pour tenir compte de la particularité de la situation des femmes au Burundi, il a consacré une direction générale à la promotion de la femme et du genre (art 3 du Décret no 100/57 du 4 avril 2016). De plus, le ministère a développé de nombreux programmes 
visant à remettre en question la discrimination basée sur le genre. On peut citer à titre d'exemple le projet d'urgence relatif aux violences sexuelles et basées sur le genre et la santé des femmes dans la région des Grands Lacs financé au Burundi par la Banque Mondiale en juin 2018.

En plus de ces structures, le gouvernement du Burundi a créé le Forum National des Femmes par le décret no 100/306 du 21 novembre 2012. Cet organe constitue un cadre d'expression des femmes de toutes les couches sociales sur tous les sujets intéressant leur vie et leurs milieux. Le décret qui définit ses missions lui reconnaît le droit de proposer ses aspirations sur toutes les questions de promotion et de protection des droits de la femme (Décret no 100/026 du 15 février 2017 art 10). La composition de cet organe reflète le souci d'inclusion de toutes les couches de femmes dans l'avancement de leur droit. Les membres sont recrutés au niveau collinaire, communal, provincial et national. Cette représentativité élargie parait être un gage de prise en compte des situations variées affectant les droits de la femme (Décret no 100/026 du 15 février 2017 art 4). Pour opérationnaliser les actions du ministère ayant en charge les questions des droits humains, le gouvernement a initié la création des Centres de Développement Familial et Communautaire (CDFC) (Décret no 100/325 du 17 décembre 2012). Cet organe décentralisé jusqu'au niveau communal traite de toutes les questions qui rentrent dans le mandat du ministère de tutelle (art 1 du Décret no 100/325 du 17 décembre 2012). Aux termes de l'article 6, le texte créant ces centres traite spécifiquement de la promotion des droits des femmes et de l'égalité de genre. Les CDFC sont ainsi habilités à «promouvoir l'égalité des genres en milieu rural et urbain » et à «mettre en application les résolutions et recommandations issues des conférences régionales et mondiales sur la femme et l'égalité des sexes ratifiées par le Burundi » (art 6 al 2 et 4 du Décret no 100/325 du 17 décembre 2012).

En dépit de ces efforts, du chemin reste à parcourir pour endiguer le phénomène de la stigmatisation de la femme infertile au Burundi, le travail en synergie entre l'administration à la base et les mouvements associatifs féminins ont un rôle important à jouer dans ce combat.

\section{III.3.2 Les efforts des mouvements associatifs}

Le paysage burundais des droits humains s'est considérablement enrichi avec la création des associations ou d'autres organisations qui œuvrent pour améliorer la prise en compte et le respect des droits de l'homme. On observe une prise de conscience croissante au haut niveau dans le combat contre ce phénomène encore tabou dans la société burundaise. C'est dans ce cadre que s'inscrit le lancement en avril 2019, du partenariat entre la Fondation Merck et la fondation Ubuntu. Cette collaboration en association avec le Ministère de la Santé et le Gouvernement du Burundi vise à développer des 
programmes pour combattre ce fléau. L'initiative «Merck» vise à autonomiser les femmes infertiles à travers l'accès à l'information, à l'éducation et à la santé et le changement de mentalités. Cette initiative aide les gouvernements à définir des politiques visant à améliorer l'accès à des soins de fertilité réglementés, sûrs et efficaces. (Kadri et al, 2005). Cet engagement au plus haut niveau est un indicateur que les plus hautes autorités prennent finalement conscience de la réalité de la stigmatisation de la femme infertile et de l'importance du combat à mener pour l'éradiquer. Dans ce cadre partenarial, les Fondations Ubuntu et Merck participent dans la sensibilisation du grand public en organisant des formations à l'intention des professionnels des médias burundais sur leur rôle à briser la stigmatisation autour de l'infertilité et des femmes infertiles. À ces diverses initiatives s'ajoute un mouvement associatif féminin (CAFOB, Dusherehamwe.) très développé qui contribue à la conscientisation des victimes et de la communauté sur les méfaits de la stigmatisation. Ces actions concourent à promouvoir l'émergence du leadership féminin et la pleine participation des femmes dans la vie civile, politique et économique de la nation.

En plus des fondations, la défense des droits humains fait partie du champ d'intervention de plusieurs organisations de la société civile (Association pour la Paix et les Droits de l'Homme, Association des femmes juristes du Burundi, Action des Chrétiens pour l'Abolition de la Torture au Burundi, association Dushirehamwe, Synergie des partenaires pour la promotion des droits de la femme, etc.). Parfois, ces organisations de la société civile opèrent sous forme de collectif pour accroitre la portée de leurs travaux ou pour plus d'efficacité dans l'élaboration des projets qu'ils soumettent à leurs bailleurs (collectif des Associations et ONGs Féminines du Burundi et Fédération nationale des Associations engagées dans le Domaine de l'Enfance au Burundi). Ces organisations sont pour la plupart des ONG qui interviennent de manière générale sur des thématiques diverses en matière de droit de l'homme. Parmi leurs missions, on peut citer à titre d'exemple celle d'amener les communautés, spécialement les femmes au maintien de la paix à la transformation sociale et à la défense des droits de la personne humaine.

Avec l'appui de l'ONU Femmes, l'objectif par l'association Dushirehamwe a mis sur pied sur toute l'étendue du pays des représentantes femmes et filles (Abakanguriramahoro= les femmes à la recherche de la paix) avec les domaines spécifiques d'interventions entre autres : la prévention et la résolution des conflits, le dialogue communautaire des femmes et filles dans la communauté, et l'autonomisation des femmes. Certes les ONG locales constituent une catégorie d'acteurs très importants dans les efforts de prévention et de réponses aux violences basées sur le genre. Elles restent incontournables puisqu'elles sont issues de la population et sont mieux acceptées. Toutefois, leurs capacités opérationnelles, notamment en termes de 
développement de projet, de ressources financières, de ressources humaines, de suivi et évaluation, restent limitées et ne garantissent pas toujours la pérennité des actions entreprises, surtout lorsque celles-ci dépendent principalement de financements de la communauté internationale. Outre la nécessité de renforcer leurs capacités institutionnelles et techniques, le gouvernement devrait investir davantage dans les institutions étatiques à l'échelle provinciale, territoriale et locale.

Ainsi, les femmes « actrices de la paix et de dialogue » sont dans toutes les provinces du pays. Ils disposent de 158 formatrices en genre et Transformation des conflits; de 390 femmes leaders spécialisées dans le rapprochement et la réconciliation communautaire et de 352 groupements comptant plus de 10000 membres servant à la base pour alerte précoce, la médiation et la dénonciation des violences faites aux femmes. Ainsi, comme réalisations, chaque année, le réseau des femmes «abakanguriramahoro» contribue à la résolution de 7000 conflits dans la communauté.

On estime que ces initiatives ne peuvent être prises pour produire seulement un effet d'annonce et témoigner d'une sympathie de façade. De ce passage, on retient qu'au Burundi, il se dessine une évolution réglementaire et institutionnelle favorable à l'effectivité de la femme. Mais, en traitant cette question de stigmatisation, on risque d'être emporté par la passion que par la raison. Si la solution durable à cette question traine, c'est un retard attribuable au décalage entre l'évolution des mentalités et le rythme d'accélération dans la mise en place des outils institutionnelle et réglementaire. Il parait alors que c'est ce temps d'ajustement qui suscite les impatiences alors que dans les outils de programmation à long terme, cette question de l'appui à la défense des droits des victimes des violations des droits humains en l'occurrence celui de la stigmatisation des femmes infertiles est prise en compte. Certes, ces efforts sont louables, mais ils restent des initiatives à promouvoir surtout au niveau de l'administration locale.

\section{III.4 Propositions d'amélioration des dispositifs mis en place par rapport à la protection de la femme infertile stigmatisée}

Le Conseil communal, l'Administrateur communal ainsi que le Chef de colline ou de quartier constituent ce qu'il convient de qualifier d'administration locale. Ces autorités de l'administration à la base disposent d'un cahier de charge qui renferme des obligations sur lesquelles s'articulent leurs actions quotidiennes. Le dispositif de ce document administratif comporte un volet concernant les questions sociales en général, et en particulier les questions relatives à la vie des ménages et des couples. Les autorités à la base peuvent se servir de cette tribune pour insérer les mesures de lutte contre le phénomène de stigmatisation dans leurs interventions (Kagisye, 2009). 
Pour renforcer son efficacité et ses interventions, l'administration locale devrait se servir du PND qui est désormais un instrument de planification à l'échelle nationale. D'introduction récente, cet outil contient des références et des indications précises sur les actions susceptibles d'améliorer la prise en charge du problème de stigmatisation. Dans son Axe d'Orientation no 13 sur la Gouvernance, le PND prévoit de consolider l'état de droit et les droits humains (PND 2018-2027). Pour matérialiser cet objectif, le PND propose des projets de renforcer les capacités des acteurs étatiques et non étatiques en matière des droits humains. Ce plan de développement envisage des projets pouvant appuyer la défense des droits des victimes des violations des droits humains (PND 2018-2027). L'administration locale peut alors se servir de ce cadre d'orientation sur la planification nationale, pour mettre en place des projets qui cadrent avec les priorités de développement économique et social du pays.

Sous le poids de la culture burundaise et des coutumes défavorables au statut socioculturel de la femme stigmatisée, celle-ci n'arrive pas à dénoncer sa situation parce que débattre de l'infertilité dans la communauté reste tabou. Pour briser ce silence, l'administration locale pourrait créer des centres d'écoute animés par des professionnels qui aideraient les victimes à sortir de leur angoisse. Pour l'efficacité de ces structures, l'administration devrait s'appuyer sur des actions de formation des parties prenantes et renforcer ellemême ses capacités d'intervention. Le combat contre le phénomène de stigmatisation de la femme infertile exige des interventions préventives qui exigent à leur tour des structures appropriées. La mise en place des comités locaux d'alerte pourrait contribuer à la réussite des efforts engagés pour la lutte contre le phénomène. Il est aussi possible d'étendre le mandat des comités ou autres structures existants pour insérer ces thématiques de stigmatisation de la femme infertile dans leurs champs d'intervention. Sur base des indicateurs d'alerte et de prévention préétablis, au niveau communautaire, ces structures rapporteraient aux centres d'écoute tout soupçon de stigmatisation. Les interventions de ces centres et les actions de sensibilisation permettraient de maîtriser des barrières socioculturelles qui handicapent la dénonciation des cas de violence surtout dans les ménages.

Les différents organes en charge de la promotion des droits humains devraient travailler en synergie et l'administration locale devrait prendre le devant pour coordonner les efforts entrepris et soutenir les actions des structures ainsi mises en place. Pour éviter de naviguer à vue, l'administration locale devrait profiter suffisamment de cadre légal existant qui pourtant était prometteur pour combattre ce phénomène.

\section{Conclusion}

L'infertilité constitue un problème social aux retombées négatives sur 
les couples et l'harmonie sociale. La femme infertile subit des stigmatisations ayant des conséquences qui ruinent l'estime de soi et réduit la femme à un être inférieur. Cette marginalisation impose à la victime une situation d'injustice qui aboutit au rejet de la femme par sa belle-famille et parfois par son conjoint.

L'étude a montré que le Burundi a manifesté son attachement à régler la question de l'injustice sociale en posant les bases juridiques pour lutter contre la discrimination basée sur tout type d'exclusion sociale. La solennité de cette adhésion est manifestée dans la loi fondamentale qui dispose que «les droits et devoirs proclamés et garantis, entre autres, par la Déclaration universelle des droits de l'homme, les Pactes internationaux relatifs aux droits de l'homme, la Charte africaine des Droits de l'Homme et des peuples, la Convention sur l'élimination de toutes les formes de discrimination à l'égard des femmes et la Convention relative aux droits de l'enfant font partie intégrante de la Constitution de la République du Burundi» (art 19 Constitutions du 7 juin 2018). Le cadre institutionnel et les instruments de programmation à long terme tel le PND et la PNG constituent aussi des références pour mener la lutte contre la stigmatisation. Ces avancées légales et institutionnelles mises en place par le gouvernement sont certes loin de bouleverser la coutume qui considère toujours à tort la femme comme la seule responsable de l'infertilité dans un couple. Cependant, les efforts entrepris pour combattre le phénomène commencent à produire des effets même s'ils sont à leur stade embryonnaire. Les dénonciations publiques des autorités au haut niveau montrent que la question de la stigmatisation est prise au sérieux. Cette prise de conscience constitue un appui qu'il faut mettre à contribution pour intensifier les actions en développant de nouvelles initiatives ensemble avec tous les intervenants.

Cette étude soutient que les efforts entrepris pour traiter le problème de stigmatisation pour cause d'infertilité de la femme sont satisfaisants, mais ne sont pas suffisants. Pour leur amélioration continue, cette réflexion considère que l'administration locale est la pierre angulaire dans la lutte contre le phénomène de stigmatisation. Ainsi propose-t-elle de créer des centres d'écoute ou d'étendre le mandat des structures existantes pour y inclure la prise en charge des femmes victimes de stigmatisation. Pour accroitre l'efficacité de ces centres ou autres structures, cette analyse suggère la mise en place des mécanismes d'alerte reposant sur des indicateurs d'intervention prétablis. L'administration locale devrait accentuer les efforts de coordination des divers intervenants pour plus d'efficacité.

\section{References :}

1. Abraham N. et al. (2005). "Psychological distress among suffering from couple infertility in south Africa: a quantitative assessment". Human reproduction, Volume 20, issue 7. 
2. Amandine R. (2015) "Les porteurs de stigmates. Entre expériences intimes, contraintes institutionnelles et expressions collectives ", Sociologie [En ligne], mis en ligne le 17 décembre 2015. http://journals.openedition.org/sociologie/2701/. Consulté le 12 Avril 2020.

3. Chateauneuf D. "Projet familial, Infertilité et désir d'enfant : usages et expériences de la procréation médicalement assistée en contexte Québécois". Diffusion numérique. Mise en ligne le 2 mars 2012. https://www.erudit.org/fr/revues/efg/1900-v1-n1efg030/1008146ar/plan/. Consulté le 16 Avril 2020.

4. Courtwright A. (2013). "Stigmatisation and Public Health Ethics". Journal of Bioethical Inquiry.

5. Deslaurier C. (2003/4). "Le «bushingantahe» peut-il réconcilier le Burundi" ? Politique africaine (No 92).

6. Dupiech S. (2004). Usagères des nouvelles technologies de la reproduction dans un contexte de communauté virtuelle. L'Harmattan, Paris.

7. Dyer S.I et al. (2005). "Psychological distress among women suffering from couple infertility in south Africa: a quantitative assessment". Human reproduction, Volume 20. (No 92).

8. Erny P. (1968). L'enfant dans la pensée traditionnelle de l'Afrique Noire. Payot, Paris.

9. Erving G. (1963). Stigmate. Les usages sociaux des handicaps. Les Editions de minuit, Paris.

10. Erving G. (1975). Stigmate. Editions de Minuit, Paris.

11. Greil A. (1991). "Not Yet Pregnant: Infertile Couples in Contemporary America, New Brunswick". NJRutgers University Press in Google Scholar.

12. Hakizimana A. (2002). Naissances au Burundi entre tradition et planification. L'Harmattan, Paris.

13. Kagisye E. (2009). La résolution des conflits de compétences entre les acteurs de la justice de proximité au Burundi, Mémoire défendu en vue de l'obtention du DESS en Droits de l'Homme et résolution pacifique de conflits, Bujumbura.

14. Laely T. (1992). 'Le destin du Bushingantahe : transformations d'une structure locale d'autorité au Burundi ", Genève-Afrique, 30 (2), pp. 75-98.

15. Larsen U. (1994). "Sterility in Sub-saharan Africa", Population studies, vol. $48, \mathrm{n}^{\circ} 3.4$

16. Makobero D. (2001). "L'institution des Bashingantahe comme moyen de réconciliation ", Au cour de l'Afrique, 1-2. 
17. Maggio C. (2006). Femme infertile. Image de soi et désir d'enfant. In Press Edition, Paris.

18. Nana P.N. et al. (2011). "Aspects psycho-sociaux chez les patients infertiles à la Maternité Principale". Mother and child health, Vol. 8.

19. Navas J. et al. (1977). Famille et fécondité au Burundi. Approche sociologique, Presse Lavigerie, Bujumbura.

20. Niyongere J. (2009). Les incidences de la stérilité sur le vécu psychosocial des vieilles personnes, Mémoire de fin d'études en vue de l'obtention du diplôme de licence en Psychologie et des Sciences de l'Education, étude menée en Mairie de Bujumbura, Université du Burundi.

21. Nkurunziza F. (2009). L'analyse psychosociale de la vie relationnelle entre une femme sans enfant et sa belle-mère, Mémoire présenté pour l'obtention du grade de licencie en Psychologie Clinique et Sociale, Université du Burundi.

22. Ntabona A. (1985). "Le concept de l'Umushingantahe et ses implications sur l'éducation de la jeunesse d'aujourd'hui au Burundi ", Au Cour de l'Afrique, 5.

23. Olatunji A. et al. (2003). "The pattern of infertility cases at a University Hospital" in Wajm Vol.22 $\mathrm{n}^{\circ} 3$.

24. Ombelet W. (2012). "Global access to infertility care in developing countries: a case of human rights, equity and social justice", issues in ObGyn.

25. Poncelet C. et al. (2011). Physiologie, Pathologie, Thérapie de la Reproduction chez l'humain, Springer Verlag, Paris.

26. Rodegem F. M. (1996). "Structures judiciaires traditionnelles au Burundi ", Revue juridique de droit écrit et coutumier du Rwanda et du Burundi, 6 (1).

27. Rostaing C. (2015). "Stigmate ", Sociologie. https://journals.openedition.org/sociologie/2572/ Consulté le 14 Juin 2020.

28. Thornicroft G. et al. (2007). "Stigma : Ignorance, prejudice". The British Journal of Psychiatry 190(3):192-3.

29. Accord d'Arusha pour la Paix et la Réconciliation au Burundi signé le 28 août 2000.

30. Décret no 100/57 du 4 avril 2016, portant missions, organisation et fonctionnement du ministère des droits de la personne humaine des affaires sociales et $\mathrm{du}$ genre. Disponible sur http://www.presidence.gov.bi/wp-content/uploads/2017/04/decret057-2016.pdf/. (page consultée le 15 Mai 2020 à 13 h 10).

31. Décret no 100/325 du 17 décembre 2012 portant création, structure, missions et fonctionnement des centres de développement familial et 
communautaire (CDFC) au ministère de la Solidarité nationale, des droits de la personne humaine et du genre (Burundi). Disponible sur http://www.presidence.gov.bi/wp-content/uploads/2017/04/decret057-2016.pdf/. (page consultée le 15 Mai 2020 à 9 h 30).

32. Décret no 100/050/97 du 21 mars 1997 portant nomination des membres du Conseil des bashingantahe pour l'unité nationale et la réconciliation (Burundi). Disponible sur http://www.presidence.gov.bi/wp-content/uploads/2017/04/decret057-2016.pdf/. (page consultée le 10 Mai 2020 à 17 h 40).

33. Décret no 100/026 du 15 février 2017 portant révision du décret no 100/306 du 21 novembre 2012 portant création, missions, organisation et fonctionnement du forum national des femmes au Burundi, article 4. Disponible sur http://www.presidence.gov.bi/wpcontent/uploads/2017/03/Decret-026-2017.pdf. (page consultée le 19 Mai 2020 à $11 \mathrm{~h}$ ).

34. Loi $\mathrm{n}^{\circ} 1 / 13$ du 22 septembre 2016 portant prévention, protection des victimes et répression des violences basées sur le genre.

35. Méthode documentaire. En ligne: https://bu.univ-avignon.fr/wpcontent/uploads/2013/08/Methodo_documentaire.pdf/. (page consultée le 9 Septembre 2020 à 21 h 20).

36. Plan d'Action 2017-2021 de Mise en œuvre de la Politique nationale Genre 2012 -2025.

37. Politique nationale de Développement du Burundi 2017-2025.

38. Rapport de l'Organisation Mondiale de la Santé 2013. https://www.who.int/whr/fr./ (page consultée le 28 Juin 2020 à 10 h). 